\title{
A Routability-Driven Complimentary-FET (CFET) Standard Cell Synthesis Framework using SMT
}

\author{
Chung-Kuan Cheng, Chia-Tung Ho, Daeyeal Lee, Dongwon Park \\ University of California, San Diego, CA, US \\ ckcheng@ucsd.edu,c2ho@ucsd.edu,ldaeyeal@ucsd.edu,dwp003@ucsd.edu
}

\begin{abstract}
As the technology node is evolving, standard cell (SDC) design scaling is obstructed by design constraints such as limited routing resources, lateral $\mathrm{P}-\mathrm{N}$ separation, and performance requirements. Complimentary-FET (CFET) technology, which stacks the P-FET on $\mathrm{N}-\mathrm{FET}$ or vice versa, is able to release the restriction of $\mathrm{P}-\mathrm{N}$ connection for SDC layout scaling. However, (both in-cell and block-level) routable CFET SDC design, while maintaining the scaling advantages, is a non-trivial problem because of the extremely limited routability (including pin-accessibility) comes from the intrinsic stacked FET structure.

In this paper, we propose an SMT (Satisfiability Modulo theories)based framework to automate CFET SDC synthesis through the simultaneous place-and-route optimization methodology with a novel Dynamic Complimentary Pin Allocation scheme. Moreover, our framework generates optimized CFET SDC layouts in terms of routability through our novel pin access and routing resource related objectives/constraints, while the scaling advantage of CFET structure is maintained compared to conventional FET structure. We demonstrate that CFET cell structure provides $10.1 / 22.2 \%$ on average reduced cell width and metal length compared to conventional FET structure. Moreover, we validate that our routability-driven features successfully improve routability in practical circuit designs through block-level analysis. Compared to the previous work, our routability-driven framework improves $4.2 \%$ utilization and reduces $83 \%$ routing errors on average in block-level designs.
\end{abstract}

\section{INTRODUCTION}

As the technology is scaling beyond $7 \mathrm{~nm}$, cell layout scaling of conventional (Conv.) FET structure is limited due to the routing congestion, the lateral $\mathrm{P}-\mathrm{N}$ separation, and performance requirements. Complimentary-FET (CFET) technology, which stacks the $\mathrm{P}-\mathrm{FET}$ on $\mathrm{N}-\mathrm{FET}$ or vice versa, can release in-cell routing congestion of P-N connection such that SDC designers continue the cell size reduction in sub- $7 \mathrm{~nm}$. Figure 1 shows an illustration of Conv. cell structure and CFET cell structure which stacking P-FET on N-FET. Compared to Conv. cell architecture, the shared or split Gate and S/D (G/S/D) structure provides flexible local interconnect connection ${ }^{1}$.

\footnotetext{
${ }^{1}$ If the G/S/D of P-FET and N-FET share the same net connection, the G/S/D can be merged and connect to the $M 0$. On the contrary, the G/S/D are split and $M 0$ drops tall
} and short vias to connect P-FET and N-FET, respectively.

Permission to make digital or hard copies of all or part of this work for personal or classroom use is granted without fee provided that copies are not made or distributed for profit or commercial advantage and that copies bear this notice and the full citation on the first page. Copyrights for components of this work owned by others than ACM must be honored. Abstracting with credit is permitted. To copy otherwise, or republish to post on servers or to redistribute to lists, requires prior specific permission and/or a fee. Request permissions from permissions@acm.org.

ICCAD '20, November 2-5, 2020, Virtual Event, USA

(c) 2020 Association for Computing Machinery.

ACM ISBN 978-1-4503-8026-3/20/11...\$15.00

https://doi.org/10.1145/3400302.3415611

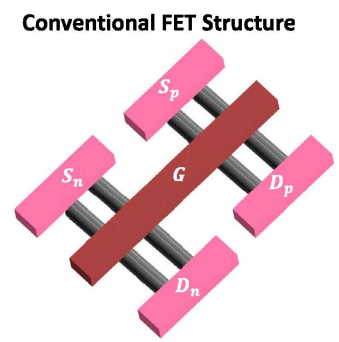

Complimentary-FET (CFET) Structure
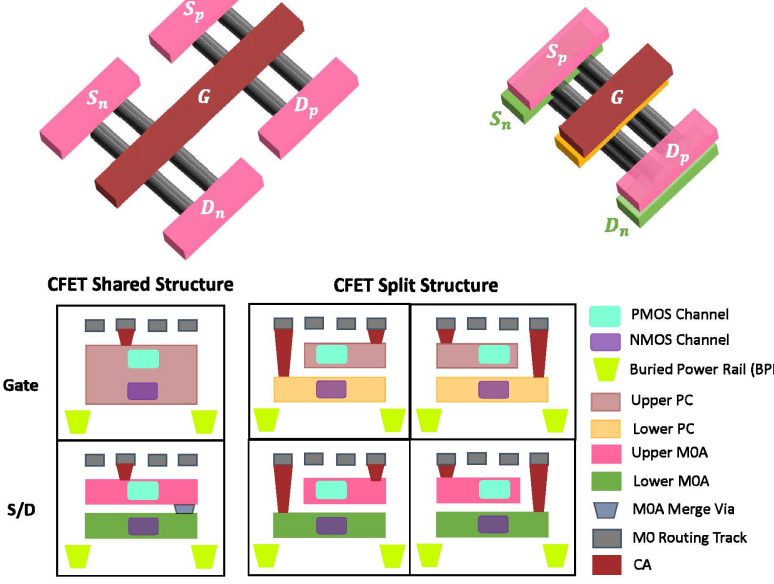

Figure 1: An illustration of Conventional and Complimentary FET (CFET) structure (Top Row). CFET shared and split Gate, Source and Drain structure (Bottom Row) [20, 22].

Recently, feasible CFET-based SDC layouts are successfully proposed [20,22], therefore CFET has been one of the promising cell structure in sub-7 $\mathrm{nm}$ or beyond. However, the SDC design in sub$7 \mathrm{~nm}$ demands holistic exploration (including block-level analysis) in terms of pin-accessibility and routing congestion due to the limited routing resource and exploded conditional design rules of later physical design procedures. This exploration for SDC design relies on automatic SDC layout synthesis.

SDC Synthesis Automation. In [9, 25], authors reported full automation of cell layout covering transistor-level placement and in-cell routing together, but these approaches are not applicable in the multi-patterning technologies in sub-5nm. For multi-patterning technology nodes, $[7,10,12]$ proposed SDC synthesis automation, but the placement and routing are performed in separate operations. Recently, in [15], they integrate the placement and routing with dynamic pin allocation (DPA) interface using Satisfiability Modulo theories (SMT) [1]. However, these works focus on the Conv. FET cell structure optimization thus it is not available in CFET cell structure due to the stack-able P/N-FET structure.

Pin-accessibility-driven Cell Layout. Several approaches have attracted considerable attention to improve the pin-accessibility of SDCs in advanced node technology [18, 19, 23, 24]. However, these approaches depend on solving sub-problems therefore it is hard to reach the optimal solution of SDC layout because of the intractable search space partitioning and the intrinsic limitation of heuristic methodology. The [4] suggested strict constraint-based pin-accessibility ensuring methodologies such as Minimum I/O Pin Length (MPL) and Minimum I/O Pin Opening (MPO). However, holistic pin-accessibility between placed SDCs is not proved through block-level analysis.

In this paper, we propose a SDC synthesis automation framework for CFET by a dynamic pin shape/allocation scheme, resulting in 


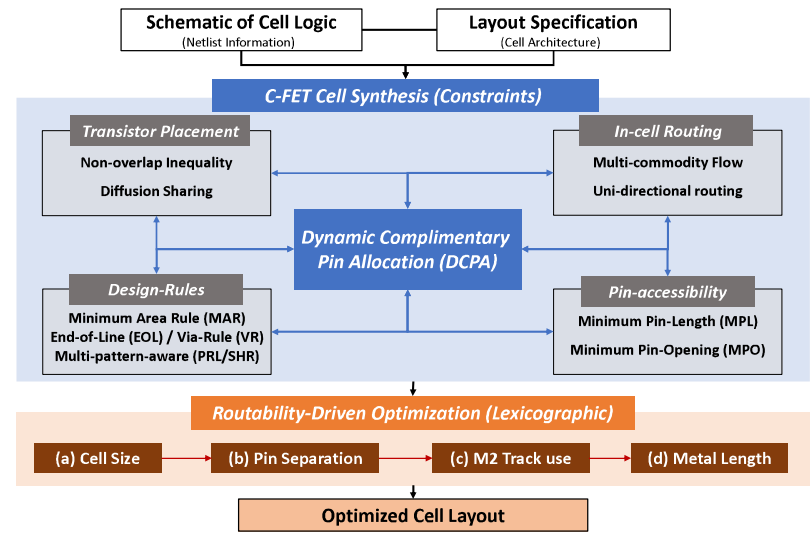

Figure 2: Framework Overview

optimized cell layout considering various design considerations. Moreover, our SDC layout has maximized routability (not only pinaccessibility) through integrated guarantees in our objectives and constraints. Our main contributions are as follows.

- We construct a framework to automate the CFET SDC physical synthesis including concurrent transistor placement and in-cell routing through a novel Dynamic Complimentary Pin Allocation (DCPA) scheme.

- We formulate an integrated constraint satisfaction problem (CSP) for SMT (Satisfiability Modulo theory) solving, including not only place-and-route but also pin-accessibility and design rule related constraints, resulting in the optimized cell layout across whole considerations.

- We propose routability-driven process including strict constraintbased MPL and MPO, objective-based pin separation (pinaccessibility), and objective-based M2 track use (routing congestion), resulting in a routability-driven SDC layout.

- We validate our framework through the block-level analysis including the \#DRV analysis across suggested design features. This empirical results prove that our objectives successfully improve the routability through multi-objective optimization.

The remaining sections are organized as follows. Section 2 describes our CFET SDC synthesis framework. Section 3 discusses our experiments. Section 4 concludes the paper.

\section{SIMULTANEOUS PLACE-AND-ROUTE FOR CFET SDC SYNTHESIS FRAMEWORK}

We utilize an SMT (Satisfiability modulo theories)-based constraints solving methodology for simultaneous place and route of CFET SDCs. In this section, we describe the detailed features of our framework: (i) Overview of CFET SDC Synthesis Framework, (ii) CFET Cell Architecture, (iii) Dynamic Complimentary Pin Allocation (DCPA), and (iv) Routability-Driven Cell Optimization.

\subsection{CFET SDC Synthesis Framework Overview}

Figure 2 shows the overview of our framework. Given cell netlist and layout specification, our framework formulates an integrated constraint satisfaction problem (CSP) so that the automated cell layout simultaneously/strictly satisfies all constraints, which are transistor placement, in-cell routing, conditional design rules and pin-accessibility-driven constraints. Inspired by [15], individual
Placement Grid

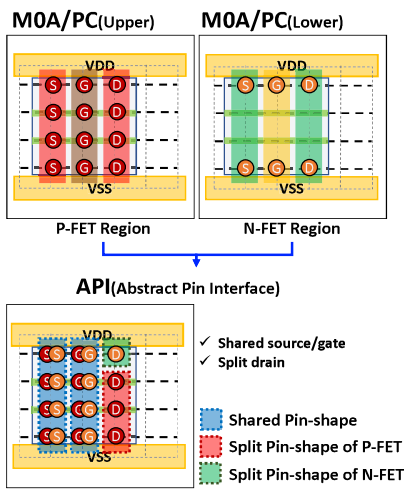

Routing Grid

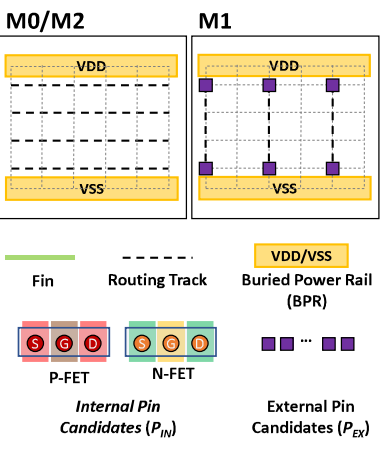

Figure 3: Grid-Based placement and routing graph with Abstract Pin Interface (API)

constraints are combined by our novel dynamic pin shape and allocation constraint (i.e., DCPA). To ensure the routability, our framework maximizes PS (Pin separation) and minimizes M2 Track use including objectives of [15] (i.e., Cell Size and Metal Length) through the lexicographic ordered optimization. We utilize five representative conditional design rules of $[13,14]$, which are minimum area rule (MAR), end-of-line (EOL), via rule (VR), and multi-patternaware design rules (i.e., PRL (Parallel Run-Length)/SHR (Step Height Rule)). The notations are shown in Table 1.

Table 1: Notations for CFET Cell Synthesis Framework

\begin{tabular}{|l||l|}
\hline \multicolumn{1}{|l|}{ Term } & Description \\
\hline$h$ & Number of M0/M2 horizontal routing tracks. \\
\hline$T$ & Set of FETs. \\
\hline$x_{t}^{p} / x_{t}^{n}$ & X coordinate of lower-left corner $t^{t} h$ P-FET/N-FET. \\
\hline$P_{E X}$ & Standard Cell I/O pins. \\
\hline$p_{i, t}^{p} / p_{i, t}^{n}$ & $i^{t h}$ pin of $t^{t} h$ P-FET/N-FET. \\
\hline$x(p)$ & $X$ coordinate of pin $p$; (i.e., $\left.x\left(p_{i, t}^{p}\right)=x_{t}^{p}+i\right)$. \\
\hline$n(p)$ & Net information of pin p. \\
\hline$d_{i n t}(k)$ & Set of M1 tracks in the pin interference distance of $k^{t h}$ M1 track. \\
\hline$G(V, E)$ & Three-dimensional $(3 \mathrm{D})$ routing graph. \\
\hline$V\left(V_{i}\right)$ & Set of vertices in $\left(i^{t h}\right.$ metal layer of) the routing graph $G$ \\
\hline$v$ or $v_{x, y, l}$ & A vertex with the coordinate $(x, y, l)$. \\
\hline$v_{d}$ & A $d$-directional ${ }^{2}$ adjacent vertex of $v$ \\
\hline$a(v)$ & Set of adjacent vertices of $v$ in $\mathrm{G}$. \\
\hline$e_{v, u}$ & An edge between $v$ and $u, u \in a(v)$. \\
\hline$w_{v, u}$ & Weighted cost for metal segment on $e_{v, u .}$. \\
\hline$n$ & $n^{t h}$ multi-pin net. \\
\hline$m$ & $m^{t h}$ sink of $n$. \\
\hline$v^{n}$ & $0-1$ indicator if $v$ is used for $n$. \\
\hline$e_{v, u}^{n}$ & $0-1$ indicator if $e_{v, u}$ is used for $n$. \\
\hline$f_{m}^{n}(v, u)$ & $0-1$ indicator if $e_{v, u}$ is used for commodity $f_{m}^{n}$. \\
\hline$m_{v, u}$ & $0-1$ indicator if there is a metal segment on $e_{v, u}$. \\
\hline$C_{m}^{n}(v, u)$ & Capacity variable for $e_{v, u}$ of commodity $f_{m}^{n}$. \\
\hline$E_{k}^{l}$ & Set of $l^{t h}$ layer edges in the $k^{t h}$ track. \\
\hline
\end{tabular}

\subsection{CFET Cell Architecture}

Our framework employs a CFET cell architecture and netlist information of [20,22] and [5], respectively. Figure 3 shows the gridbased placement and routing graph (i.e., Upper/Lower M0A/PC, Abstract Pin Interface (API), M0, M1, and M2). The routing grid consists of 4 horizontal tracks with buried power rails (i.e., 4.5 Track Height) and each layer is defined as unidirectional edges. We adopt supernodes [11] for the pin of FET (i.e. internal pin, $P_{I N}$ ) or the I/O pin of a standard cell (i.e. external pin, $P_{E X}$ ). Unlike the Conv. cell architecture, P-FET and N-FET regions are stacked up on the

${ }^{2}$ The symbol $d$ is $L$ (Left), $R$ (Right), $F$ (Front), $B$ (Back), $U$ (Up), $D$ (Down), or a combination of these directions, e.g., $F L$ means FrontLeft. 


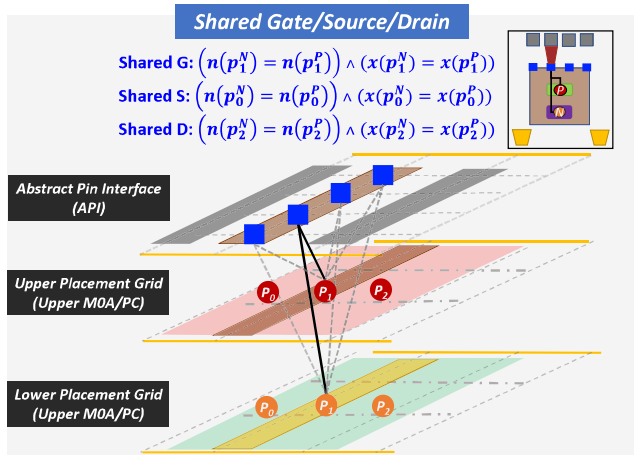

(a)
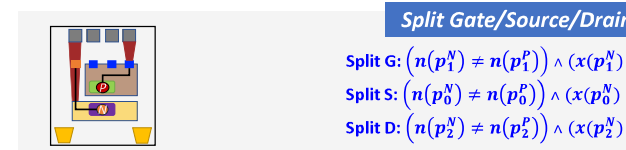

Split G: $\left(n\left(p_{1}^{N}\right) \neq n\left(p_{1}^{P}\right)\right) \wedge\left(x\left(p_{1}^{N}\right)=x\left(p_{1}^{P}\right)\right)$

Split S: $\left(n\left(p_{0}^{N}\right) \neq n\left(p_{0}^{P}\right)\right) \wedge\left(x\left(p_{0}^{N}\right)=x\left(p_{0}^{P}\right)\right)$

Split D: $\left(n\left(p_{2}^{N}\right) \neq n\left(p_{2}^{P}\right)\right) \wedge\left(x\left(p_{2}^{N}\right)=x\left(p_{2}^{P}\right)\right)$

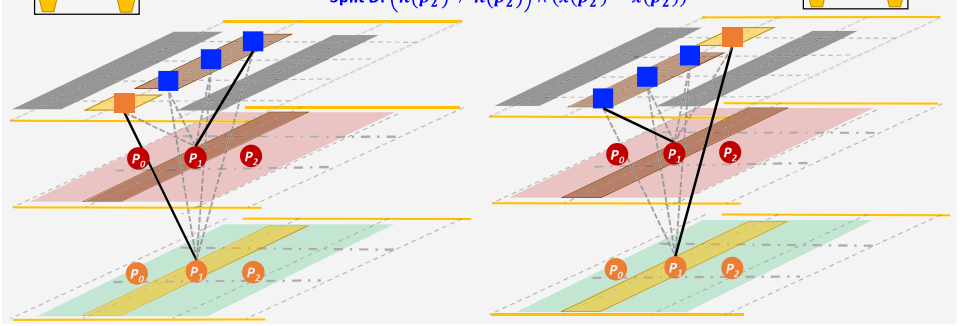

(b)

Access point for Pin P P-FET Pin (Supernode) @ N-FET Pin (Supernode)

Figure 4: Concept of Dynamic Complimentary Pin Allocation (DCPA) for CFET cell structure.

upper and the lower $M 0 A / P C$ layers, respectively. Therefore, the access to the $M 0$ layer from each pin on the N-FET region (i.e. lower $M 0 A / P C$ layer) is restricted to the top or bottom horizontal routing track unless each source/gate/drain pin in P-FET and N-FET that are overlapped on the same vertical track is shared $[20,22]^{3}$. As a result, there exist three kinds of pin shapes according to the sharing status of each pin in stacked FETs as depicted in Figure 1.

We propose the API for describing the pin-shapes on upper and lower M0A/PC layer together, resulting in reduced complexity of our framework. A novel Dynamic Complimentary Pin Allocation (DCPA) approach is devised to perform simultaneous place-androute and to select the pin shapes of FETs dynamically.

\subsection{Dynamic Complimentary Pin Allocation}

DCPA dynamically constructs the shared and split pin-shapes of FETs in the abstract pin interface (API) for an optimal in-cell routing exploration of a CFET structure. The dynamic pin allocation (DPA) scheme for simultaneous place-and-route follows the same principle of [15] for interconnecting placement and routing formulas using flow capacity variables (i.e., $C_{m}^{n}(p, r)$ ).

Figure 4 illustrates the concept of DCPA. When the pins of P-FET and N-FET are located at the same $\mathrm{x}$-coordinate (i.e., $x\left(p_{i}^{P}\right)=x\left(p_{i}^{n}\right)$ ), the pin-shape (i.e., shared or split) at the corresponding column in the API is determined by the net information. For example, in Figure 4(a), if both of the gate pins $p_{1}^{N}$ and $p_{1}^{P}$ belong to the same net (i.e., $\left.n\left(p_{1}^{N}\right)=n\left(p_{1}^{P}\right)\right)$, a shared pin-shape is selected and one of the corresponding flow variables (i.e., $f_{m}^{n}$ ) among four possible access points (i.e., blue squares) is determined by the flow formulation. On the other hand, if each gate pin belongs to the different nets (i.e., $\left.n\left(p_{1}^{N}\right) \neq n\left(p_{1}^{P}\right)\right)$, DCPA selects one of two possible split pin-shapes (i.e., top or bottom access point for N-FET) as shown in Figure 4(b). Meanwhile, the split pin-shape with bottom N-FET access point is always selected when the P-FET has a connection to the power rail (i.e., VDD) due to the CFET's buried power rail structure. The split structure is used for inactive source and drain pins of FETs.

Algorithm 1 utilizes SMT's if-then-else structure to describe a generation procedure of the constraint for our novel dynamic pinshape selection scheme. When N-FET and P-FET pins share the same location $x(p)$, the net information $n(p)$ is used to determine the corresponding pin-shape in API. The flows of each N-FET and P-FET pin are set to 1 for the corresponding access points of each

${ }^{3}$ In our paper, we adopt the stacked structure of [22], which is the P-FET on N-FET, to describe our approach in later sections. Our framework can easily support stacking $\mathrm{N}$-FET on P-FET by swapping the FET-related variables.

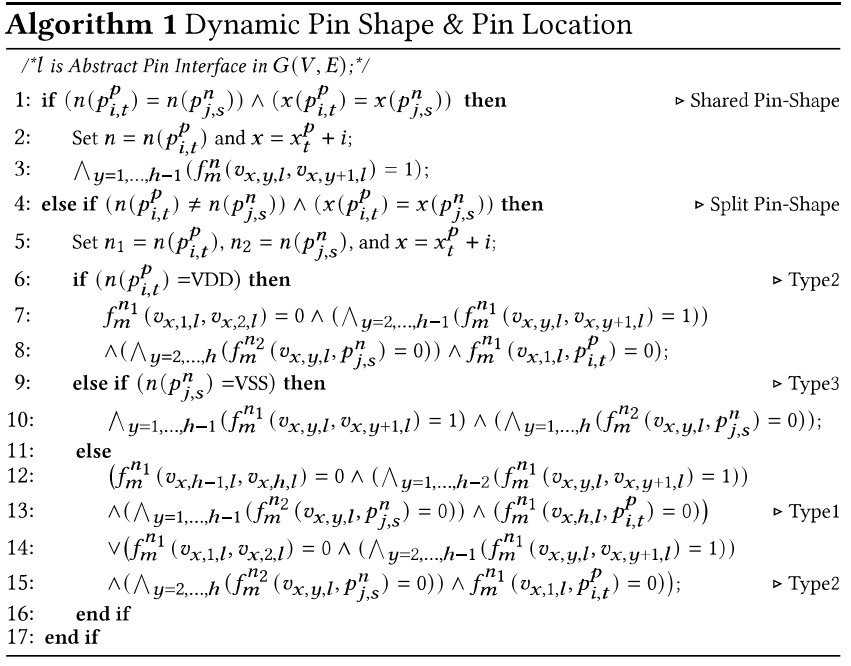

pin-shape. If N-FET and P-FET pins have the same net information, the shared pin-shape is selected (Lines 1-3). Otherwise, the split pin-shape is selected (Lines 4-16). The split pin-shape consists of three types. Type 1 and Type 2 represent top $(y=h)$ and bottom $(y=1)$ accesses for N-FET, respectively. Type 3 represents that there is no connection from M0 to N-FET pin. If the net of P-FET pin is VDD, Type 2 is always selected (Lines 7-8). If the net of N-FET pin is VSS, Type 3 is selected for P-FET pin accesses (Lines 10). Otherwise, Type 1 or Type2, which satisfies all the constraints and produces the optimal solution, is selected (Lines 12-15).

\subsection{Routability-Driven Cell Optimization}

For routability, we propose strict constraint-based pin-accessibility improvement methods; (i) minimum I/O pin opening (MPO) and (ii) minimum I/O pin length (MPL). Also, we suggest new objectives, (i) pin separation (PS) and (ii) M2 track use, to improve the pinaccessibility and the routing resource congestion, respectively.

2.4.1 Constraint-based Pin-Accessibility Improvement: Our framework utilizes MPL and MPO constraints to improve pin accessibility as [4] suggested (but, details were not addressed).

MPL (Minimum I/O Pin Length). MPL rule defines the minimum number of metal segments of the commodity heading to the external pin $P_{E X}$ on the $M 1$ layer as shown in Figure 5(a). At-least 1 (AL1) metal segment on the $M 1$ layer must be assigned to the commodity whose sink is $P_{E X}$ as expressed in Constraint (1). Then, the metal 
segment on the $M 1$ layer is extended to the minimum length defined by MAR. The vertices on the extended segments are the possible pin access points.

$$
\begin{aligned}
& \operatorname{AL1}\left(m_{v, v_{F}}, m_{v, v_{B}}\right), \quad \text { if } f_{m}^{n}\left(v, v_{D}\right)=1, f_{m}^{n}\left(v, v_{U}\right)=1 \\
& \forall v \in V_{1}, m=P_{E X}
\end{aligned}
$$

MPO (Minimum I/O Pin Opening). MPO rule ensures the minimum number of unblocked access points (i.e., pin openings) from the $M 2$ layer for each I/O pin. Figure 5(b) illustrates that each pin candidate $v_{p}$ has to secure enough horizontal space on the $M 2$ layer so that it can be accessed through the $M 2$ layer without violating design rules such as the MAR and EOL. MPO considers each $v_{p}$ as the possible pin opening if there is no routed metal segment in the opening mask (depicted in light yellow rectangles) on the M2 layer. MPO is a boolean cardinality constraint to ensure at-least-k (ALk) true pin opening indicator $O_{v_{p}}$ among the possible candidates $Q(p)$ as described in Constraint (2). If there exist any edges $e_{v, u}^{n}$ on the M2 layer, MPO is not applied because the external pin $p$ already has unblocked access points on the M2 layer.

$$
\operatorname{ALk}\left(\left\{O_{v_{p}} \mid v_{p} \in Q(p)\right\}\right), \quad \text { if } \bigvee_{v \in V_{2}, u \in V_{2}} e_{v, u}^{n(p)}=0
$$

For the example of Figure 5(b), the $O_{v_{p}}$ is set as 1 (true) if there is no routed metal segment in the opening mask, whose length is the summation of MAR and EOL parameters (i.e., MAR $+2 \times \mathrm{EOL}$ ), as shown in Constraint (3).

$$
\begin{aligned}
\overline{O_{v_{p}}}= & \sum_{n \in N, n \neq n(p)}\left(\left(e_{v_{L L}, v_{L}}^{n} \vee e_{v_{L}, v_{p}}^{n} \vee e_{v_{p}, v_{R}}^{n}\right) \wedge\left(e_{v_{L}, v_{p}}^{n} \vee e_{v_{p}, v_{R}}^{n} \vee e_{v_{R}, v_{R R}}^{n}\right)\right), \\
& \forall v_{p} \in Q(p), \quad\left\{\begin{array}{ll}
v \in Q(p), & \text { if } e_{v_{D}, v_{D F}}^{n}=1, e_{v_{D}, v_{D B}}^{n}=1 \\
v \notin Q(p), & \text { otherwise }
\end{array}, \forall v \in V_{2}\right.
\end{aligned}
$$

2.4.2 Objective-based Pin-Accessibility Improvement (Pin Separation): We propose a Pin Separation (PS) objective, which counts the number of $\mathrm{I} / \mathrm{O}$ pins that keep the minimum spacing (i.e., $d_{\text {int }}[19]$ ) from each other. Then we maximize the total number to disperse the pins as many as possible. Figure 6 illustrates the different pinaccessibility according to the spacing between pins. Figure 6(a) and (b) have the I/O pins with spacing of 0 and 1 , respectively. When the $M 2$ wires are accessing the left and right pins, the access points of the center pin in Figure 6(a) become in-accessible. In contrast, the center pin in Figure 6(b) maintains its accessibility. This shows that as the more pins are scattered across a cell, the more access points are available.

2.4.3 Objective-based Routing-Congestion Minimization (M2 track use): We set a $\# M 2$ track objective, which counts the number of occupied $M 2$ tracks in a cell, and minimize it to suppress the routing congestion because the M2 layer is jointly used in both front-end and back-end layout design. Figure 7 illustrates the impact of the different M2 layer blockages on the routing congestion. The I/O

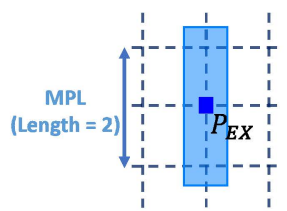

(a)

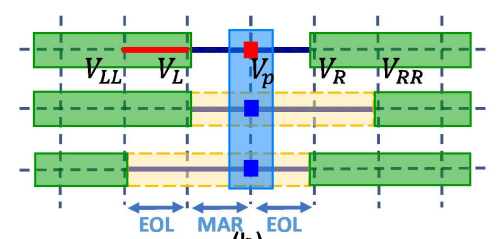

(b)
Opened Access Point $\square$ Blocked Access Point $\square$ M1 $\square$ M2

Figure 5: An example of (a) MPL with MAR=2, (b) MPO with EOL/MAR = 1/1.

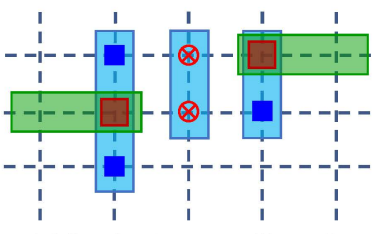

(a) Spacing between Pins $=0$

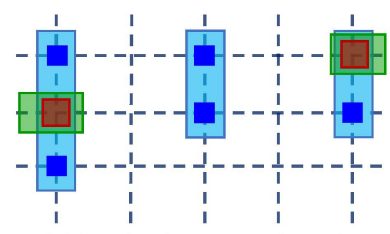

(b) Spacing between Pins = 1
Accessible Point $\otimes$ In-Accessible Point

M1 $\square$ M2 $\square$ VIA

Figure 6: An illustration of Pin Separation (PS)

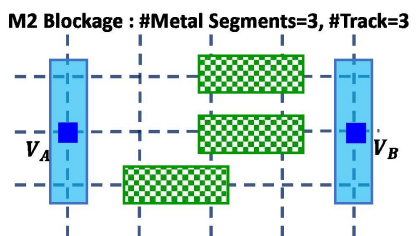

(a) Detoured Connection $\left(V_{A} \leftrightarrow V_{B}\right)$

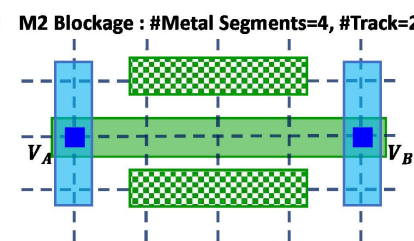

(b) Direct Connection $\left(V_{A} \leftrightarrow V_{B}\right)$
口 I/O Pins with Same Net $\quad 8$ M2 Blockage $\square$ M1 $\square$ M2

Figure 7: An illustration of the Impact of M2 Blockage on the Routing Congestion.

pin $V_{A}$ has a connection to pin $V_{B}$. The $M 2$ blockages in Figure 7(a) has metal segments $=3$ and occupies 3 routing tracks. The $M 2$ blockages in Figure 7(b) has metal segments $=4$, but, occupies 2 routing tracks. As a result, the connection in Figure 7(b) can be directly routed, while the connection in Figure 7(a) must have a detour in the routing path. This demonstrates that the number of occupied $M 2$ tracks has more impact on the routing congestion than the length of $M 2$ metals.

2.4.4 Multi-objective Optimization (Optimal Priority): Our framework has multiple objectives associated with placement and routing problems for standard cell layout design. The first objective is cell size which is defined as the right-most vertical track as shown in Expression (4). The second objective is Pin separation (PS) and it is defined as the number of pins that have no adjacent pins within an interference distance $d_{\text {int }}$ (Expression (5)). The third objective is the number of $M 2$ tracks used for in-cell routing (Expression (6)). The last objective is the weighted sum of routed metal segments (i.e., Total Metal Length (ML)) as shown in Expression (7). In practice, the cell size has the highest priority because it has a direct impact on the area of a whole chip. The PS should be considered as the second objective because the in-accessible pins can not be routed regardless of the routing resources. Then the number of $M 2$ tracks has been used as a more important metric than Total ML to maximize the routability by reserving upper routing resources. Therefore, our framework simultaneously optimizes these multiple objectives based on addressed "lexicographic" order (Expression (8)) through an optimization feature of OMT[1].

Minimize: Placement (Cell Size) $=\max \left\{x_{t}+w_{t} \mid t \in T\right\}$

Maximize: Pin-accessibility (Pin Separation) $=\sum_{p \in P_{E X}} S(p)$

$$
S(p)=\bigwedge_{e_{v, q} \in E_{k}^{M 1}, k \in d_{i n t}(x(p)), q \in P_{E X}, q \neq p} \neg e_{v, q}^{n(q)}
$$

Minimize: Routability (\#M2 Track) $=\sum_{k=1}^{h} \bigvee_{e_{v, u} \in E_{k}^{M 2}} m_{v, u}$ 


$$
\text { Minimize: Total ML }=\sum_{e_{v, u} \in E}\left(w_{v, u} \times m_{v, u}\right)
$$

Lexicographic Optimization:

(a)CellSize, (b)PinSeparation, (c)\#M2Track, (d)TotalML

$(8)$

\section{EXPERIMENTAL RESULTS}

Our framework is implemented in Perl/SMT-LIB 2.0 standardbased formula and validated on a workstation with $2.4 \mathrm{GHz}$ Intel Xeon E5-2620 CPU and 256GB memory. The single-threaded SMT Solver Z3 [1] (version 4.8.5) is used to produce the optimized solution through the proposed framework. We use ASAP7[5] SDC SPICE netlists as inputs of CFET SDCs. We adopt the same FET width and number of fingers from [5] for SDC layout generation in the following experiments. Note that 30 representative cells, which are specified in Table 2, are selected for all experiments. The experiment parameters of conditional design rules $[13,14]$ are as follows: MAR/EOL/VR/PRL/SHR $=1 / 1 / 1 / 1 / 2^{4}$.

\subsection{CFET vs. Conv. Cell Layout}

In this section, we demonstrate the optimized XOR2x1 CFET cell layout using our DCPA scheme and compare the cell width, total metal length, and \#M2 Tracks of CFET SDCs with Conv. SDCs. The Conv. SDCs are generated using the framework in [15]. For the fair comparison in terms of our metrics, we adopt the same in-cell horizontal routing tracks (i.e., 4 tracks) and push the SDC power rail to BPR layer for Conv. SDC structure [17].

Figure 8 shows the netlist and the generated CFET cell layout of an XOR2x1 SDC. The shared and split pin-shapes have been successfully selected by our DCPA scheme. When the source and drain of each FET on the same column have the same net information, the shared pin-shapes are selected (depicted in columns 3,12 ). If the net information is different, the split pin-shapes are selected and the locations (i.e., top or bottom) of N-FET access points are determined by DCPA. If the source or drain of each FET have no connection to VDD or VSS, the top (Type1, depicted in columns 17) or bottom (Type2, depicted in columns 21) track is selected as an access point of N-FET. For the P-FET with VDD connection and N-FET with VSS connection, Type2 (depicted in columns 15, 19) and Type 3 (depicted in column 5) are respectively selected.

Table 2 enumerates the comparison results of CFET SDCs and Conv. SDCs. The number of FETs in each cell varies from 2 to 24 and the average runtime per cell is less than 7 minutes. Compared to the Conv. SDCs, the CFET SDCs achieves $10.1 \%, 22.2 \%$, and $22.5 \%$ reduction on the average cell width, metal length, and \#M2 tracks,

${ }^{4}$ We assume that the VR of CA layer allows at most two diagonal vias in the experiments.

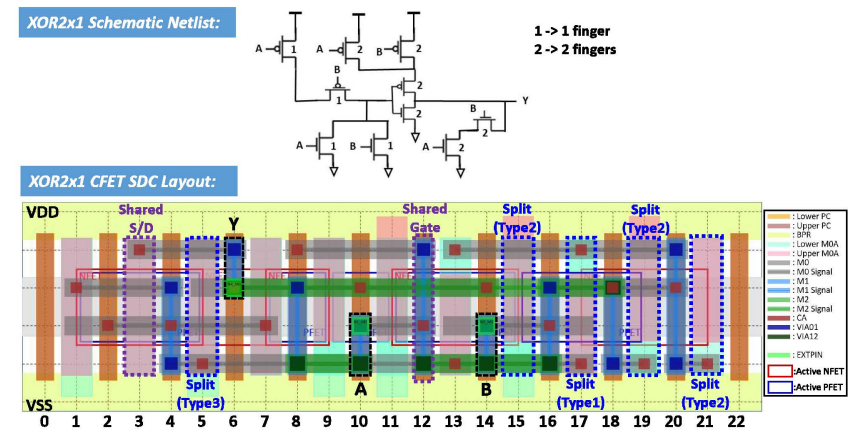

Figure 8: An example of XOR2x1 Schematic Netlist [5] and CFET SDC Layout.
Table 2: Experimental Statistics: $\mathbf{M L}=$ Metal Length. $\mathbf{C P P}=$ Contact Poly Pitch. Cell Width Red. $=(($ Cell Width of reference - Cell Width of CFET $) /$ Cell width of reference $)$. ML Red. = ((ML of reference $-M L$ of CFET $) / M L$ of reference $)$.

\begin{tabular}{|c|c|c|c|c|c|c|c|c|c|c|c|c|}
\hline \multicolumn{3}{|c|}{ Cell Specification } & \multicolumn{9}{|c|}{ Cell Layout Metric } & \multirow{3}{*}{ Runtime(s) } \\
\hline \multirow{2}{*}{ Name } & \multirow{2}{*}{ \#FET } & \multirow{2}{*}{ \#Net } & \multicolumn{3}{|c|}{ Cell Width (CPPs) } & \multicolumn{3}{|c|}{ Metal Length } & \multicolumn{3}{|c|}{ \#M2 Tracks } & \\
\hline & & & Conv. & CFET & Red.(\%) & \begin{tabular}{|l|} 
Conv. \\
\end{tabular} & CFET & Red.(\%) & Conv. & CFET & Red.(\%) & \\
\hline AND2x2 & 6 & & 7 & 6 & 14.3 & 80 & 54 & 32.5 & 0 & 0 & & 4.6 \\
\hline AND3x1 & 8 & 9 & 6 & 6 & & 87 & 62 & 28.7 & 0 & 0 & & 97.3 \\
\hline AND3x2 & 8 & 9 & 8 & 7 & 12.5 & \begin{tabular}{l|l}
102 \\
\end{tabular} & 70 & 31.4 & 0 & 0 & & 18.0 \\
\hline AOI21x1 & 6 & 8 & 10 & 9 & 10.0 & 372 & 393 & -5.7 & 3 & 4 & -33.3 & 170.4 \\
\hline AOI22x1 & 8 & 10 & 15 & 14 & 6.7 & 556 & 501 & 9.9 & 3 & 3 & & 464.9 \\
\hline BUFx2 & 4 & 5 & 6 & 5 & 16.7 & \begin{tabular}{l|l|l}
67 & \\
\end{tabular} & 42 & 37.3 & 0 & 0 & & 1.6 \\
\hline BUF $x 3$ & 4 & 5 & 7 & 6 & 14.3 & 90 & 51 & 43.3 & 0 & 0 & & 3.1 \\
\hline BUFx4 & 4 & 5 & 8 & 7 & 12.5 & \begin{tabular}{l|l}
97 \\
\end{tabular} & 56 & 42.3 & 0 & 0 & & 4.4 \\
\hline BUFx8 & 4 & 5 & 13 & 12 & 7.7 & 203 & 101 & 50.3 & 0 & 0 & & 21.5 \\
\hline DFFHQN & 24 & 17 & 19 & 18 & 5.3 & 616 & 300 & 51.3 & 2 & 1 & 50.0 & $1,959.9$ \\
\hline FA & 24 & 17 & 16 & 15 & 6.3 & 641 & 396 & 38.2 & 3 & 2 & 33.3 & $5,688.5$ \\
\hline INVx1 & 2 & 4 & 3 & 3 & & 31 & 22 & 29.0 & 0 & 0 & $t$ & 0.9 \\
\hline INVx2 & 2 & 4 & 5 & 4 & 20.0 & \begin{tabular}{l|l}
49 \\
\end{tabular} & 26 & 46.9 & 0 & 0 & & 0.7 \\
\hline INVx4 & 2 & 4 & 7 & 6 & 14.3 & \begin{tabular}{l|l|}
76 \\
\end{tabular} & 50 & 34.2 & 0 & 0 & & 1.6 \\
\hline INVx8 & 2 & 4 & 11 & 10 & 9.1 & \begin{tabular}{l|l|}
140 \\
\end{tabular} & 89 & 36.4 & 0 & 0 & & 4.7 \\
\hline NAND2x1 & 4 & 6 & 6 & 6 & & \begin{tabular}{l|l|}
117 \\
\end{tabular} & 80 & 31.6 & 1 & 0 & 100.0 & 2.4 \\
\hline NAND2x2 & 4 & 6 & 13 & 10 & 23.1 & \begin{tabular}{l|l}
208 \\
\end{tabular} & 170 & 18.3 & 1 & 1 & & 9.6 \\
\hline NAND3x1 & 6 & 8 & 12 & 11 & 8.3 & 207 & 279 & -34.8 & 1 & 2 & -100.0 & 54.6 \\
\hline NAND3x2 & 6 & 8 & 25 & 21 & 16.0 & \begin{tabular}{l|l}
680 \\
\end{tabular} & 790 & -16.2 & 3 & 3 & & $1,161.6$ \\
\hline NOR2x1 & 4 & 6 & 6 & 6 & - & 124 & 79 & 36.3 & 1 & 0 & 100.0 & 8.9 \\
\hline NOR2x2 & 4 & 6 & 13 & 10 & 23.1 & \begin{tabular}{l|l|}
305 \\
\end{tabular} & 150 & 50.8 & 2 & 0 & 100.0 & 41.4 \\
\hline NOR3x1 & 6 & 8 & 12 & 11 & 8.3 & 209 & 205 & 1.9 & 1 & 0 & 100.0 & 81.4 \\
\hline NOR3x2 & 6 & 8 & 26 & 22 & 15.4 & \begin{tabular}{l|l|}
610 \\
\end{tabular} & 372 & 39.0 & 1 & 1 & & 276.7 \\
\hline OAI21x1 & 6 & 8 & 11 & 11 & & 479 & 391 & 18.4 & 4 & 3 & 25.0 & 188.9 \\
\hline OAI22x1 & 8 & 10 & 15 & 15 & & |567 & 564 & 0.5 & 3 & 3 & & 284.0 \\
\hline OR2x2 & 6 & 8 & 7 & 6 & \begin{tabular}{l|l|}
14.3 \\
\end{tabular} & 80 & 53 & 33.8 & 0 & 0 & & 6.2 \\
\hline OR3x1 & 8 & 9 & 6 & 6 & & \begin{tabular}{l|l}
87 \\
\end{tabular} & 61 & 29.9 & 0 & 0 & & 120.6 \\
\hline OR3x2 & 8 & 9 & 8 & 7 & 12.5 & 100 & 70 & 30.0 & 0 & 0 & & 44.6 \\
\hline XNOR2x1 & 10 & 9 & 12 & 12 & & 510 & 456 & 10.6 & 3 & 3 & & 626.5 \\
\hline XOR2x1 & 10 & 9 & 13 & 12 & 7.7 & \begin{tabular}{|l|l}
548 \\
\end{tabular} & 322 & 41.2 & \begin{tabular}{l|l}
4 \\
\end{tabular} & \begin{tabular}{l|l}
2 & \\
\end{tabular} & 50.0 & \begin{tabular}{|l|l|}
139.1 \\
\end{tabular} \\
\hline Avg. & \begin{tabular}{|l|l|}
6.8 \\
\end{tabular} & 7.7 & \begin{tabular}{l|l|}
10.9 \\
\end{tabular} & 9.8 & \begin{tabular}{|l|}
10.1 \\
\end{tabular} & \begin{tabular}{|l|}
267.9 \\
\end{tabular} & \begin{tabular}{|l|}
208.5 \\
\end{tabular} & 22.2 & 1.2 & 0.9 & 22.5 & 382.9 \\
\hline
\end{tabular}

respectively. Figure 9 shows a design-rule corrected DFFHQN cell layout for Conv. and CFET architectures. All metal segments that are depicted in red dashed rectangles are successfully extended to satisfy conditional design rules such as PRL and SHR. By virtue of directed P-N connections between stacked FETs, DFFHQN with CFET consumes less routing resources $(51.3 \%$ and $50.0 \%$ of metal length and \#M2 tracks, respectively) and 1 smaller $\mathrm{CPP}$ of cell width than with Conv. cell structure.

\subsection{Routability-driven Cell Optimization}

In this section, we validate our routability-driven constraints and objectives using the statistics of cell metrics, pin-accessibility metrics, \#M2 tracks, and M2 metal length with multiple CFET SDC sets generated by our framework.

3.2.1 Optimization for Pin-Accessibility. Figure 10 shows the different I/O pin distributions by PS objective for a NAND2x1 cell with design parameters, MPO $=2$ and $d_{\text {int }}=1.5 \mathrm{M} 1$ pitch. The MPL and MPO constraints respectively ensure at-least one $M 1$ metal segment and at-least 2 pin-openings for each $\mathrm{I} / \mathrm{O}$ pin (depicted in blue dashed rectangles). Though the number of pin-openings is the same for each I/O pin, the PS cost of each cell is different. While the pins of the "MPO Only" cell layout are concentrated in a certain region (Figure 10(a)), the pins of the "PS with MPO" cell layout are distributed keeping the minimum distance from each other (Figure 10(b)). The $\mathrm{RPA}^{5}$ value of each pin in "MPO Only" cell layout is smaller (i.e., worse) than that of the cell layout with PS. In particular, the RPA value of the pin $B$ without PS is 0.33 . This means that the $\operatorname{pin} B$ is not likely to be accessed successfully, because we need at least one access point. On the contrary, all the pins with PS have the same RPA value with the number of pin-openings. This demonstrates that the PS optimization efficiently improves the pin-accessibility by taking the full advantages of the MPO constraint.

${ }^{5}$ The RPA of [19] indicates how many access points of a pin remain after the accesses of its neighboring pins. 

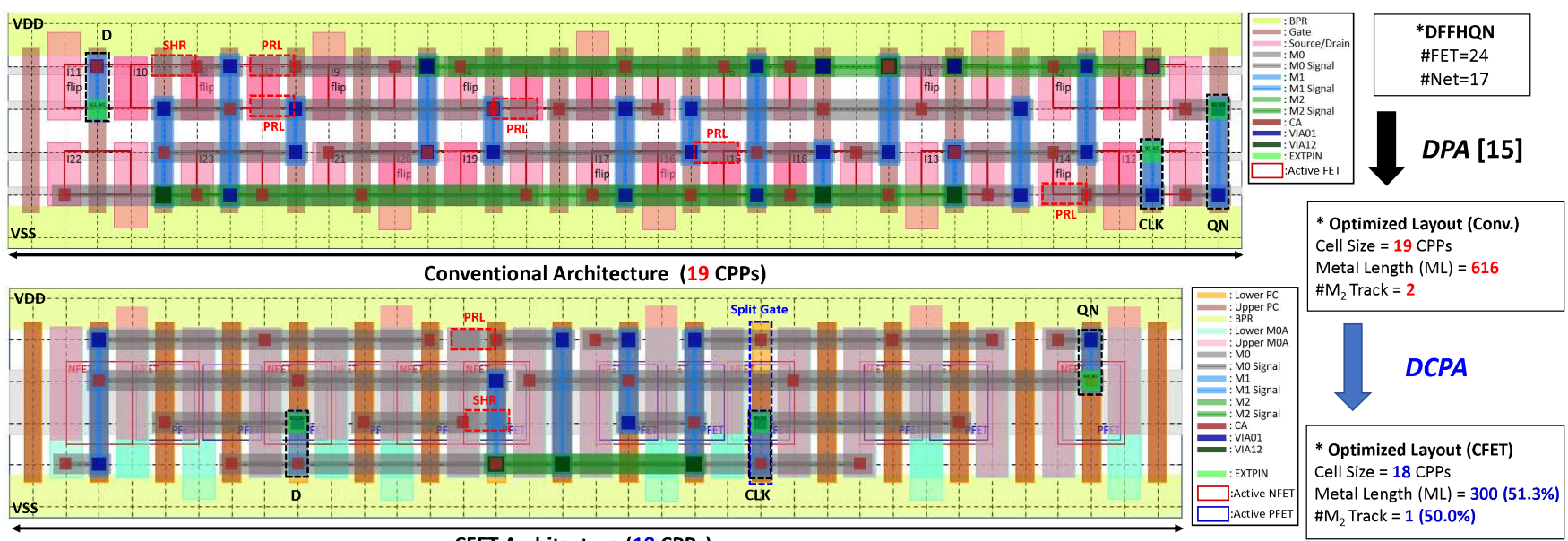

CFET Architecture (18 CPPs)

Figure 9: Layouts of 4 routing tracks CFET and Conv. DFFHQN with corrected design constraints. Optimized result of CFET layout: Cell Size (19->18), Metal Length (616->300), \#M2 Tracks (2->1). The red dash-line boxes are metal extension for PRL and SHR design constraints.

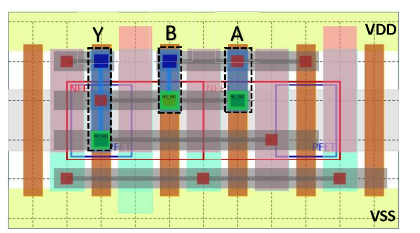

(a) MPO Constraint Only

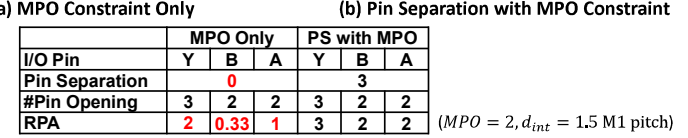

Figure 10: Layout of NAND2x1 cell optimized for Pin Separation objective.

Table 3 shows the comparison of key metrics for the split cases of MPO constraint and PS objective. "MPO=2 wPS" denotes that the SDCs are generated with the objectives including PS (i.e., the same objectives with Expression (8)) and the MPO parameter = 2. "woPS" refers to the objectives without PS (i.e., (a)CellSize, (b)\#M2Track and (c)TotalML). The Min.\#PO values, which refers to the minimum of pin-openings in each SDC, shows that our cell layout is successfully ensured \#PO by suggested MPO constraint. The average PS-objective values of "wPS" cases are $1.54 \times$ and $1.91 \times$ larger than "woPS" cases for MPO = 2 and 3, respectively. This shows that our PS objective effectively dispersed the I/O pins for each SDC. The Min.RPA value represents the minimum accessible pin-openings of generated CFET SDCs. "wPS" cases have more accessible pinopenings than "woPS" cases by $10.8 \%$ and $15.8 \%$ for "MPO=2" and "MPO $=3$ ", respectively. This demonstrates that the MPO constraint successfully ensured the minimum number of pin-openings and the PS objective contributed to maximize the effective pin-openings. As the MPO increases and the PS is maximized, the average ML and \#M2Track are increased due to the enlarged and scattered $\mathrm{I} / \mathrm{O}$ pins.

3.2.2 Optimization for Routing-Congestion Minimization. We compare the $M 2$ routing-resource related metrics between our proposed \#M2Track and M2 Length objectives, which are discussed in Section. 2.4.3, as described in Table 4. "MinTrack" denotes that the SDCs are generated with the objectives including \#M2Track (i.e., Expression (8)). "MinLength" is M2 Length-oriented objectives (i.e., (a)CellSize, (b)PS, (c)M2 Length, and (d)TotalML). Compared
Table 3: Experimental Results of 30 CFET SDCs with Pin Separation (PS) Objective Function under $\mathrm{MPO}=2$ and $\mathrm{MPO}=3$ Constraints: All values are averages, ML $=$ Total Metal Length, \#M2Track = the number of used M2 tracks, Min. $\# P O=$ the minimum of pin-openings in a cell, Min.RPA $=$ minimum remaining pin access [19], RPA impr. = improvement ratio (wPS-woPS)/woPS, interference distance $d_{\text {int }}=1.5 \mathrm{M1}$ pitch $((2 \mathrm{MAR}+\mathrm{EOL}) / 2)$, opening mask for $M P O=3(2 \mathrm{EOL}+1 \mathrm{MAR})$

\begin{tabular}{|l|r|r|r|r|r|r|r|}
\hline \multirow{2}{*}{ Settings } & \multicolumn{4}{|c|}{ Cell Metrics } & \multicolumn{4}{|c|}{ Pin-Accessibility Metrics } \\
\cline { 2 - 8 } & CellWidth & ML & \#M2Track & PS & Min.\#PO & Min.RPA & RPA impr.(\%) \\
\hline MPO=2 woPS & 9.8 & 204.3 & 0.90 & 1.73 & 2.07 & 1.67 & \multirow{2}{*}{$10.8 \%$} \\
\hline MPO=2 wPS & 9.8 & 208.9 & 0.93 & 2.67 & 2.10 & 1.85 & \\
\hline MPO=3 woPS & 9.8 & 209.7 & 0.93 & 1.50 & 3.03 & 2.40 & \multirow{2}{*}{$15.8 \%$} \\
\hline MPO=3 wPS & 9.8 & 211.2 & 0.97 & 2.87 & 3.10 & 2.78 & \\
\hline
\end{tabular}

Table 4: Experimental Results of CFET SDCs optimized for MinTrack (i.e., Expression (8)) and MinLength (i.e., (a)CellSize, (b)PS, (c)M2 Length, and (d) TotalML). Incr. $=($ MinTrack - MinLength $) /$ MinLength, Red. $=($ MinLength - MinTrack)/MinLength.

\begin{tabular}{|l|r|r|r|r|r|r|}
\hline \multirow{3}{*}{ Cell Name } & \multicolumn{7}{|c|}{ Cell Layout Metric } \\
\cline { 2 - 8 } & \multicolumn{7}{|c|}{ M2 Metal Length } & \multicolumn{3}{|c|}{ \#M2 Tracks } \\
\cline { 2 - 8 } & MinLength & MinTrack & Incr. (\%) & MinLength & MinTrack & Red. (\%) \\
\hline AOI21x1 & 34 & 34 & - & 4 & 4 & - \\
\hline AOI22x1 & 38 & 38 & - & 3 & 3 & - \\
\hline DFFHQN & 4 & 6 & 50.0 & 2 & 1 & 50.0 \\
\hline FA & 24 & 28 & 16.7 & 3 & 2 & 33.3 \\
\hline NAND2x2 & 2 & 2 & - & 1 & 1 & - \\
\hline NAND3x1 & 22 & 28 & 27.3 & 2 & 2 & - \\
\hline NAND3x2 & 52 & 64 & 23.1 & 4 & 3 & 25.0 \\
\hline NOR3x2 & 6 & 8 & 33.3 & 1 & 1 & - \\
\hline OAI21x1 & 26 & 26 & - & 4 & 3 & 25.0 \\
\hline OAI22x1 & 38 & 38 & - & 3 & 3 & - \\
\hline XNOR2x1 & 32 & 34 & 6.3 & 3 & 3 & - \\
\hline XOR2x1 & 18 & 18 & - & 3 & 2 & 33.3 \\
\hline \hline Avg. & 24.7 & 27.0 & 9.3 & 2.8 & 2.3 & 17.9 \\
\hline
\end{tabular}

to "MinLength", "MinTrack" reduces the M2 track usage by $17.9 \%$ with $9.3 \%$ of increment in $M 2$ length. Figure 8 and Figure 11 show the example of an XOR2x1 cell that are optimized using MinTrack and MinLength objectives, respectively. While both layouts have the same M2 metal length, the used M2 tracks of MinTrack is 1 less than MinLength. As discussed in Section. 2.4.3, we expect that MinTrack reduces the routing congestion in block-level more effective than MinLength due to the reduced M2 tracks in spite of the increased $M 2$ length. We validate this in Section. 3.3.

\subsection{Block-level Routability Analysis}

We validate our framework through a block-level analysis including the \#DRV analysis across suggested design features. The block-level 


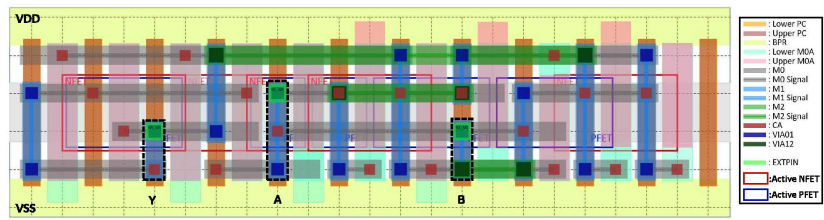

Figure 11: Layout of XOR2x1 cell optimized with MinLength objectives. Table 5: SDC Generation Split Cases for Routability Analysis

\begin{tabular}{|l|l|l|}
\hline \multicolumn{1}{|c|}{ Cases } & \multicolumn{1}{|c|}{ Objectives } & \multicolumn{1}{c|}{ Constraints } \\
\hline wPS MPO=2 (resp. 3) & (a)CellSize (b)PS (c)\#M2Track (d)TotalML & MPO=2 (resp. 3) \\
\hline woPS MPO=2 (resp. 3) & (a)CellSize (b)\#M2Track (c)TotalML & MPO=2 (resp. 3) \\
\hline MinLength & (a)CellSize (b)PS (c)M2Min (d)TotalML & MPO=2 \\
\hline MinTrack & (a)CellSize (b)PS (c)\#M2Track (d)TotalML & MPO=2 \\
\hline Proposed (best setting) & (a)CellSize (b)PS (c)\#M2Track (d)TotalML & MPO=3 \\
\hline SP\&R [15] & (a)CellSize (b)TotalML & MPO=N/A \\
\hline
\end{tabular}

analysis is executed through an industry-leading commercial tool for Place-and-Route [2].

3.3.1 Experimantal Environments. Three open source RTL designs [6], MO Core, M1 Core, and AES that respectively have $17 \mathrm{~K}, 20 \mathrm{~K}$, and $14 \mathrm{~K}$ instances are selected and the SDCs are generated on the 4.5T CFET architecture. For BEOL, we use $M 2-M 5$ for detailed routing. Firstly, we presume the pitch and width of $M 0$ and $M 2$ layer and the number of masks for each BEOL layer referring to [21]. Then, we derive the metals' pitch and width based on [16] guidance. For PDN, there are top power mesh (i.e., $M 8$ and $M 9$ ), intermediate power stripe (M3), and standard cell rails. The top power mesh is designed as dense as possible. The intermediate power stripe frequency (i.e., M3) for the BPR (Buried Power Rail) standard cell rail is 64 CPPs [3]. Then, the power is delivered from $M 3$ to $M 1$ and $M 1$ to BPR using stacked vias and SuperVia models [8], respectively.

3.3.2 Routability Analysis. We analyze the routability of three RTL designs using multiple CFET SDC sets that are generated under different split cases of pin-accessibility and routability related constraints and objectives as described in Table 5.

Analysis 1 (Pin-accessibility). Figure 12 shows the \#DRVs trends of wPS and woPS under $M P O=2$ and 3 . The \#DRVs of $M P O=3$ cases increases slower than $\mathrm{MPO}=2$ cases as the design utilization increases, because the number of pin-openings are secured as much as the MPO parameter and, also, the Min. RPAs of MPO=3 cases are both $40 \%$ larger than $\mathrm{MPO}=2$ cases (Table 3 ). The \#DRVs of wPS cases consistently grows slower than woPS cases under the same MPO for all three designs. In particular, the \#DRVs of wPS with $\mathrm{MPO}=2$ and $\mathrm{MPO}=3$ are $44 / 47 / 23 \%$ and $25 / 91 / 8 \%$ smaller than woPS with $\mathrm{MPO}=2$ and $\mathrm{MPO}=3$ at the $0.87 / 0.85 / 0.87$ utilization in M0 Core/M1 Core/AES, respectively. This demonstrates that our MPO constraint and PS objective successfully maximize the effective accessible pin-openings, resulting in the improvement of the routability.

Analysis 2 (Routing Congestion). Figure 13 shows the \#DRVs trends for MinTrack and MinLength objectives. The \#DRVs of MinTrack increases slower than MinLength objective for all designs. Specifically, the \#DRVs of MinTrack are 33/19/12\% smaller than \#DRVs of MinLength at the $0.87 / 0.85 / 0.87$ utilization in M0 Core/M1 Core/AES, respectively. Table 6 shows the pin analysis QoR reports of the commercial place-and-route (P\&R) tool [2]. The horizontal congestion of MinLength are larger than MinTrack from $0.1 \%$ up to $2.0 \%$ at each design utilization showing obvious \#DRVs differences. This validates that MinTrack is more effective objective for reducing the routing congestion than MinLength objective.
Table 6: Pin Analysis QoR Report of MinTrack and MinLength from [2]

\begin{tabular}{|l|l|r|r|r|r|r|r|}
\hline \multicolumn{2}{|c|}{} & \multicolumn{2}{|c|}{ M0 Core } & \multicolumn{2}{c|}{ M1 Core } & \multicolumn{2}{c|}{ AES } \\
\hline \multicolumn{2}{|c|}{ Utilization } & 0.87 & 0.90 & 0.91 & 0.92 & 0.87 & 0.90 \\
\hline \multirow{2}{*}{$\begin{array}{l}\text { Horizontal } \\
\text { Congestion (\%) }\end{array}$} & MinTrack & 15.5 & 35.8 & 21.9 & 30.8 & 28.3 & 33.5 \\
\cline { 2 - 8 } & MinLength & 16.9 & 36.3 & 22.4 & 32.2 & 30.3 & 33.6 \\
\hline Impr.(MinLength - MinTrack)(\%) & 1.4 & 0.5 & 0.5 & 1.4 & 2.0 & 0.1 \\
\hline
\end{tabular}

Analysis 3 (Block-level Routability). Figure 14 shows the blocklevel P\&R results of Proposed versus SP\&R [15]. Compared to SP\&R, our Proposed case shows 4.4\%, 4.0\%, and 4.1\% improvement (depicted in blue arrows) for the design utilization at 300 \#DRVs threshold of M0 Core, M1 Core, and AES. In addition, the Proposed case reduces $84 \%, 87 \%$, and $79 \%$ \#DRVs (depicted in orange arrows) of M0 Core, M1 Core, and AES at the utilization when the \#DRVs of SP \&R starts to exceed $300^{6}$ threshold line of each design. Moreover, comparing the minimum valid block-level area (i.e., \#DRVs $<300$ ) of each design, the proposed objectives further reduce the block-level area $5.7 \%, 7.3 \%$ and $6.8 \%$ of M0 Core, M1 Core and AES (depicted in purple texts), respectively. This validates that our routabilitydriven constraints and objectives not only reduce the \#DRVs but also improve the block-level area scaling. Figure 15 shows P\&R snapshots and a \#DRVs report of M0 Core at 0.82 utilization. The \#DRVs of SP\&R case is $6.4 \times$ larger than our Proposed case. Most of DRVs (depicted in white objects), which are caused by heavy routing congestion on $M 2$ layer and near the $M 3$ power stripes, have been successfully reduced in our Proposed case by improved pin-accessibility and optimized routing resources.

\section{CONCLUSION}

We propose an SMT-based CFET SDC synthesis framework with novel Dynamic Complimentary Pin Allocation scheme. For routability, the novel Pin Separation and \#M2 Track objectives with Minimum Pin Length and Minimum Pin Opening constraints are implemented and validated with the statistics of cell-level metrics and block-level routability analysis in multiple designs. We demonstrate that CFET cell structure provides $10.1 / 22.2 \%$ on average reduced cell width and metal length, maintaining the scaling advantage of CFET structure, compared to conventional FET structure with 4 incell horizontal routing tracks. The block-level routability analysis shows that our routability-driven framework improves $4.2 \%$ utilization and reduces $83 \%$ routing errors on average over the previous work [15] with 300 \#DRVs threshold.

\section{ACKNOWLEDGEMENTS}

Chung-Kuan Cheng acknowledges the support from NSF CCF1564302.

\section{REFERENCES}

[1] Nikolaj Bjørner et al. 2015. vZ-an optimizing SMT solver. In International Conference on Tools and Algorithms for the Construction and Analysis of Systems. Springer, 194-199.

[2] Cadence Innovus User Guide. 2020. http:/www.cadence.com.

[3] Bharani Chava et al. 2018. DTCO exploration for efficient standard cell power rails. In Design-Process-Technology Co-optimization for Manufacturability XII, Vol. 10588. International Society for Optics and Photonics.

[4] Chung-Kuan Cheng et al. 2020 (to appear). Standard-Cell Scaling Framework with Guaranteed Pin-Accessibility. In Proc. ISCAS. IEEE, -

[5] Lawrence T Clark et al. 2016. ASAP7: A 7-nm finFET predictive process design kit. Microelectronics fournal 53 (2016), 105-115.

[6] OpenCores: Open-Source IP Cores. 2020. https://opencores.org/.

[7] Pascal Cremer, Stefan Hougardy, Jan Schneider, and Jannik Silvanus. 2017. Automatic cell layout in the 7nm era. In Proc. ISPD. ACM, 99-106.

${ }^{6}$ From the industrial guidance, designs with \#DRVs smaller than 300 usually can be fix in Engineering Change Order (ECO) stage. 

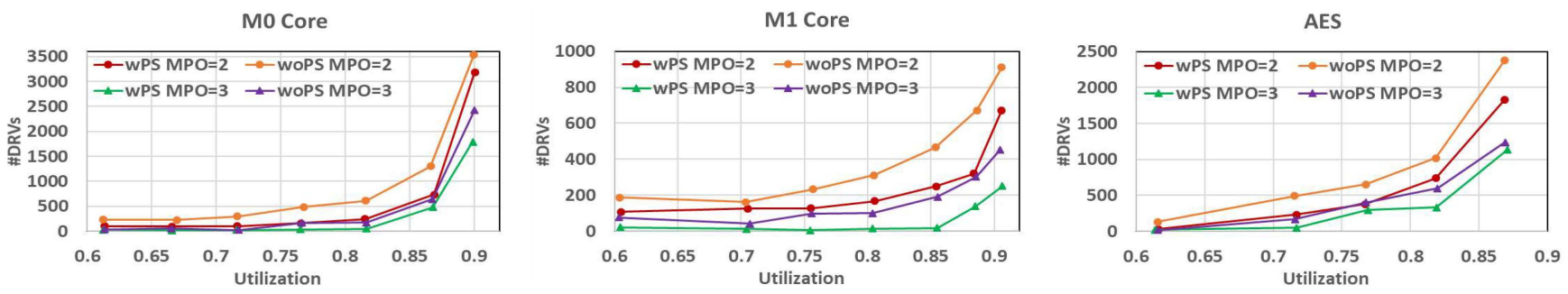

Figure 12: Block-Level Placement and Route Results of MO core, M1 core, and AES designs of wPS and woPS under MPO=2 and MPO=3 constraints.
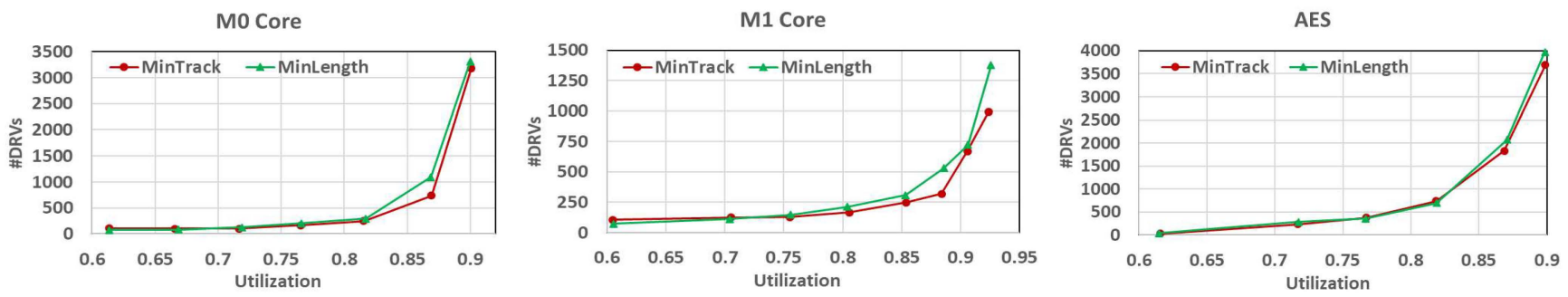

Figure 13: Block-Level P\&R Results of M0 core, M1 core, and AES designs of MinTrack and MinLength.
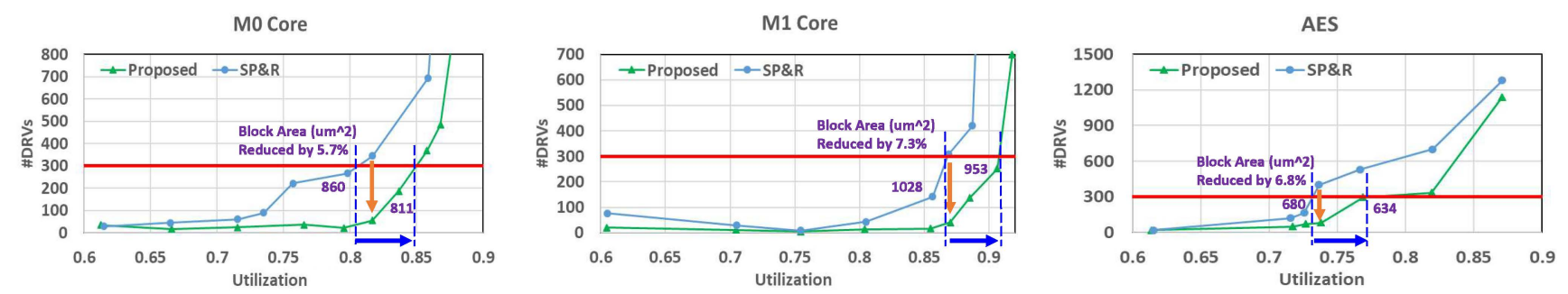

Figure 14: Block-level P\&R Results of M0 core, M1 core and AES of proposed routability-driven cell optimization versus SP\&R [15] objectives CFET SDCs.

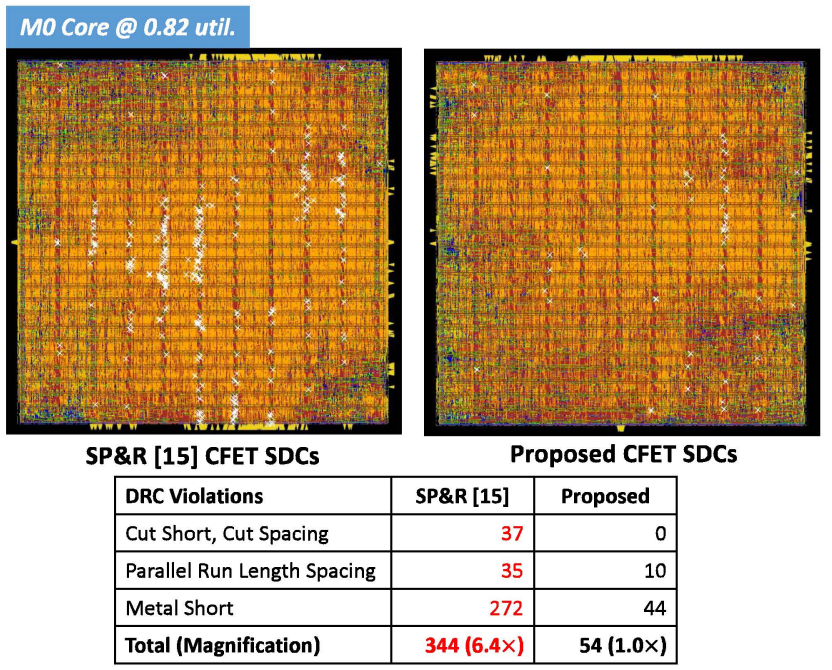

Figure 15: P\&R design views of M0 core at 0.82 util. with proposed routabilitydriven CFET SDCs versus SP\&R [15] objectives CFET SDCs. The white objects represent DRVs.

[8] Anshul Gupta et al. 2018. High-aspect-ratio ruthenium lines for buried power rail. In International Interconnect Technology Conference (IITC). IEEE, 4-6.

[9] Mohan Guruswamy et al. 1997. CELLERITY: A fully automatic layout synthesis system for standard cell libraries. In Proc. DAC. 327-332.

[10] Kyeongrok Jo et al. 2019. Design Rule Evaluation Framework Using Automatic Cell Layout Generator for Design Technology Co-Optimization. IEEE Trans. on VLSI 27, 8 (2019), 1933-1946.

[11] Ilgweon Kang et al. 2018. Fast and precise routability analysis with conditional design rules. In Proc. SLIP. ACM, 4.
[12] Yih-Lang Li et al. 2019. NCTUcell: A DDA-aware cell library generator for FinFET structure with implicitly adjustable grid map. In Proc. DAC. IEEE, 1-6.

[13] Dongwon Park et al. 2019. ROAD: Routability Analysis and Diagnosis Framework Based on SAT Techniques.. In Proc. ISPD. 65-72.

[14] Dongwon Park et al. 2020. Grid-based Framework for Routability Analysis and Diagnosis with Conditional Design Rules. IEEE Trans. on CAD (2020), 1-1.

[15] Dongwon Park et al. 2020. SP\&R: Simultaneous Placement and Routing framework for standard cell synthesis in sub-7nm. In Proc. ASP-DAC. IEEE, 345-350.

[16] LEF/DEF Language Reference. 2020. http://www.ispd.cc/contests/18/lefdefref. pdf.

[17] J Ryckaert et al. 2019. Extending the roadmap beyond 3nm through system scaling boosters: A case study on Buried Power Rail and Backside Power Delivery. In Electron Devices Technology and Manufacturing Conference (EDTM). IEEE, 50-52.

[18] Nikolay Ryzhenko et al. 2019. Pin Access-Driven Design Rule Clean and DFM Optimized Routing of Standard Cells under Boolean Constraints. In Proc. ISPD. ACM, 41-47.

[19] Jaewoo Seo et al. 2017. Pin accessibility-driven cell layout redesign and placement optimization. In Proc. DAC. IEEE, 1-6.

[20] SMY Sherazi et al. 2019. CFET standard-cell design down to 3Track height for node 3nm and below. In Design-Process-Technology Co-optimization for Manufacturability XIII, Vol. 10962. International Society for Optics and Photonics.

[21] SM Yasser Sherazi et al. 2019. Standard-cell design architecture options below 5nm node: The ultimate scaling of FinFET and Nanosheet. In SPIE. International Society for Optics and Photonics.

[22] Jeffrey Smith. 2019. Design Technology Co-Optimization Approaches for Integration and Migration to CFET and 3D Logic. In THE SURFACE PREPARATION AND CLEANING CONFERENCE (SPCC). Linx.

[23] Xiaoqing $\mathrm{Xu}$ et al. 2016. Parr: Pin-access planning and regular routing for selfaligned double patterning. Proc. ACM TODAES 21, 3 (2016), 42.

[24] Wei Ye et al. 2015. Standard cell layout regularity and pin access optimization considering middle-of-line. In Proceedings of the 25th edition on Great Lakes Symposium on VLSI. ACM, 289-294.

[25] Adriel Mota Ziesemer and Ricardo Augusto da Luz Reis. 2014. Simultaneous two-dimensional cell layout compaction using milp with astran. In 2014 IEEE Computer Society Annual Symposium on VLSI. IEEE, 350-355. 